\title{
Interferencia causada por hemólisis en la determinación de 25 constituyentes bioquímicos en el autoanalizador ADVIA 1800
}

Hemolysis interference in the determination of 25 biochemical constituents using ADVIA 1800 autoanalyzer

\author{
Ítalo Moisés Saldaña $\mathrm{O}^{1, a}$ \\ ${ }^{1}$ Departamento de Patología Clínica, Servicio de Bioquímica, Hospital Nacional Edgardo Rebagliati Martins, EsSalud, Lima, Perú. \\ ${ }^{a}$ Tecnólogo Médico- Químico farmacéutico.
}

\begin{abstract}
Resumen
Introducción. La interferencia por hemólisis es la principal causa de rechazo preanalitico de muestras de suero en el laboratorio clínico. Objetivos. Conocery cuantificar la posible interferencia producida por hemólisis en la medición rutinaria de 25 constituyentes bioquímicos en el autoanalizador ADVIA 1800, empleando para ello el criterio de interferencia clínicamente relevante, cuando se supera el máximo error sistemático deseable. Diseño. Estudio descriptivo comparativo. Institución. Hospital Edgardo Rebagliati Martins, EsSalud, Lima, Perú. Material biológico. Muestras sanguíneas proveniente de sujetos voluntarios. Intervenciones. Se añadieron cantidades crecientes de hemoglobina $(0,26 \mathrm{~g} / \mathrm{L}, 0,53 \mathrm{~g} / \mathrm{L}, 1,05 \mathrm{~g} / \mathrm{L}, 2,10 \mathrm{~g} / \mathrm{L}, 3,25 \mathrm{~g} / \mathrm{L}, 4,30 \mathrm{~g} / \mathrm{L}$ y $5,25 \mathrm{~g} / \mathrm{L})$ a siete diferentes alícuotas de una mezcla de sueros y se determinó en ellas por duplicado la influencia del interferente en los 25 constituyentes. Se siguió el protocolo de la Sociedad Española de Química Clínica. Principal medida de resultados. Porcentaje relativo de desviación de la concentración del constituyente por influencia de la hemólisis, con respecto a la muestra sin interferente. Resultados. Los constituyentes urea, creatinina, ácido úrico, bilirrubina total, colesterol HDL, colesterol LDL, triglicéridos, calcio y gammaglutamiltransferasa no presentaron interferencia, mientras que se observó interferencia para glucosa, proteínas, albúmina, colesterol, potasio, fósforo, magnesio, deshidrogenasa láctica, creatinfosfoquinasa, aspartato aminotransferasa, alanino aminotransferasa, lipasa, sodio, cloro, fosfatasa alcalina y amilasa. Conclusiones. De los 25 constituyentes estudiados, 16 presentaron interferencia clínicamente significativa. Se recomienda que cada laboratorio investigue los efectos de dicha interferencia empleando sus propios métodos, reactivos o instrumentos.
\end{abstract}

Palabras clave. Hemólisis; Interferencia por Hemólisis; Errores sistemáticos.

\section{Abstract}

Introduction. Hemolysis interference is the main cause of pre analytical rejection of serum samples in clinical laboratory. Objectives. To identify and quantify possible hemolysis interferences in the routine measurement of 25 biochemical constituents using ADVIA 1800 autoanalyzer, by clinical relevant interference criterion when the maximum desirable systematic error is exceeded. Design. Comparative descriptive study. Institution. Hospital Edgardo Rebagliati Martins, EsSalud, Lima, Peru. Biologic material. Blood samples collected from volunteer subjects. Interventions. Increasing amounts of hemoglobin $(0.26 \mathrm{~g} / \mathrm{L}, 0.53 \mathrm{~g} / \mathrm{L}, 1.05 \mathrm{~g} / \mathrm{L}, 2.10 \mathrm{~g} / \mathrm{L}, 3.25 \mathrm{~g} / \mathrm{L}$, $4.30 \mathrm{~g} / \mathrm{L}$, and $5.25 \mathrm{~g} / \mathrm{L}$ ) were added to seven different aliquots of sera mixture and influence of interfering influence in 25 constituents was determined by duplicate. The Spanish Society of Clinical Chemistry protocol was followed. Main outcome measure. Hemolysisrelated relative percentage deviation of the constituent concentration compared with the sample without interference. Results. Urea, creatinine, uric acid, total bilirubin, HDL cholesterol, LDL cholesterol, triglycerides, calcium, and gamma glutamyl transferase showed no interference. Interference was observed for glucose, protein, albumin, cholesterol, potassium, phosphorus, magnesium, lactic dehydrogenase, creatine phosphokinase, aspartate aminotransferase, alanine aminotransferase, lipase, sodium, chlorine, alkaline phosphatase and amylase. Conclusions. Out of 25 constituentss studied, 16 had clinical significant interference. It is recommended that each laboratory investigate this interference effects using their own methods, reagents or instruments.

Keywords. Hemolysis; Hemolysis Interference; Bias, Systematic.

An Fac med. 2015;76(4):377-84 / http://dx.doi.org/10.15381/anales.v76i4.11407

\section{INTRODUCCIÓN}

Las interferencias analíticas pueden causar errores clínicamente significativos en los resultados de una magnitud biológica, las cuales conducen a diagnósticos equivocados, análisis o exploraciones adicionales inapropiadas y tratamientos innecesarios o potencialmente desfavorables para los pacientes.
Se define la interferencia analítica como el efecto de un constituyente en la exactitud de medida de otro constituyente o el efecto que se produce en cualquier etapa de su determinación ${ }^{(1,2)}$.

La interferencia por hemólisis es la principal causa de rechazo preanalítico de muestras de suero, el cual conlleva a un gasto extra de reactivos, un mayor desgaste de los equipos y demora en la emisión de resultados ${ }^{(3)}$.

La prevalencia de muestras hemolizadas en diversos estudios es muy variable, oscila entre 0,05 y 3,3\%. Esta variabilidad puede deberse a las distintas formas de realizar el ensayo para investigar interferencia por hemólisis y 
a los varios criterios para establecer el límite de error máximo admisible para este tipo de interferencia ${ }^{(4,5)}$.

La calidad analítica de los resultados de un laboratorio se puede estimar por medio de indicadores como la imprecisión, el error sistemático (ES) y el error total (ET), comparándolos con las especificaciones de la calidad establecidas. El grado de cumplimiento de dichas especificaciones no solo asegura la calidad de los resultados sino que también es esencial para asegurar la interpretación y utilización de los datos de laboratorio por los clínicos.

La Organización Mundial de la Salud, con sustentación en las recomendaciones de la Sociedad Alemana de Química Clínica, define interferencia clínicamente relevante cuando se supera el error sistemático deseable ${ }^{(6)}$.

La hemólisis es el proceso de destrucción de los hematíes con la consiguiente liberación del contenido intracelular en el plasma, alterando su composición. La principal molécula intracelular es la hemoglobina, que tiene un espectro de absorción característico del grupo Hem, con un pico de $400 \mathrm{~nm}$ y varios picos entre 500 y $600 \mathrm{~nm}$, lo que produce un color rojizo en el plasma proporcional a la cantidad de hemoglobina liberada. La hemólisis puede haber sido originada in vivo por diversas alteraciones en los hematíes u otras causas, o in vitro por una extracción o manejo de la muestra de sangre inadecuada. Solo la hemólisis in vitro es considerada como interferencia ${ }^{(7,8)}$.

La hemólisis tiene un marcado efecto en aquellos analitos de elevada concentración intracelular, los cuales pueden mostrar un sesgo positivo como resultado de la liberación de estos constituyentes desde el compartimento intracelular hasta el plasma sanguíneo.

En el proceso de hemólisis también se libera líquido intracelular hacia el espacio extracelular, lo cual puede originar un sesgo negativo en ciertos constituyentes como resultado del proceso de dilución.
La hemólisis también puede producir interferencia espectral cuando la absorbancia de la hemoglobina y del cromógeno producido en la reacción se solapan, debido a que la hemoglobina tiene una elevada absorbancia, entre 400 y $600 \mathrm{~nm}$, por lo que puede interferir en procedimientos con lecturas en esa región del espectro.

Además, el grupo hemo, el hierro, la adenilato cinasa, las proteasas y otros componentes intracelulares liberados en el proceso de hemólisis, pueden interferir en diversas reacciones químicas ${ }^{(7,8)}$.

\section{MÉTODOS}

Los 25 constituyentes investigados fueron valorados en el analizador ADVIA 1800 con reactivos y calibra-

Tabla 1. Métodos y concentración sérica de los constituyentes investigados.

\begin{tabular}{|c|c|c|}
\hline Constituyente & Método & $\begin{array}{l}\text { Concentración sérica del } \\
\text { constituyente en estudio }\end{array}$ \\
\hline Glucosa & Hexocinasa & $6,66 \mathrm{mmol} / \mathrm{L}(120 \mathrm{mg} / \mathrm{dL})$ \\
\hline Urea & Ureasa con GLDH & $10,79 \mathrm{mmol} / \mathrm{L}(65 \mathrm{mg} / \mathrm{dL})$ \\
\hline Creatinina & Creatininasa & $128,18 \mu \mathrm{mol} / \mathrm{L}(1,45 \mathrm{mg} / \mathrm{dL})$ \\
\hline Ácido úrico & Uricasa/peroxidasa & $255,76 \mu \mathrm{mol} / \mathrm{L}(4,3 \mathrm{mg} / \mathrm{dL})$ \\
\hline Proteínas & Biuret & $58,1 \mathrm{~g} / \mathrm{L}(5,81 \mathrm{~g} / \mathrm{dL})$ \\
\hline Albúmina & Verde de bromocresol & $28 \mathrm{~g} / \mathrm{L}(2,8 \mathrm{~g} / \mathrm{dL})$ \\
\hline Bilirrubina total & Oxidación por vanadato & $10,43 \mu \mathrm{mol} / \mathrm{L}(0,61 \mathrm{mg} / \mathrm{dL})$ \\
\hline Colesterol total & CHOD,Trinder & $3,57 \mathrm{mmol} / \mathrm{L}(138 \mathrm{mg} / \mathrm{dL})$ \\
\hline HDL colesterol & Eliminación/catalasa. Directo & $0,85 \mathrm{mmol} / \mathrm{L}(32,75 \mathrm{mg} / \mathrm{dL})$ \\
\hline LDL colesterol & Eliminación/catalasa. Directo & $2,25 \mathrm{mmol} / \mathrm{L}(87 \mathrm{mg} / \mathrm{dL})$ \\
\hline Triglicéridos & GPO, Trinder sin blanco de suero & $1,55 \mathrm{mmol} / \mathrm{L}(137 \mathrm{mg} / \mathrm{dL})$ \\
\hline Sodio & Potenciometría indirecta & $133 \mathrm{mmol} / \mathrm{L}(133 \mathrm{mEq} / \mathrm{L})$ \\
\hline Potasio & Potenciometría indirecta & $3,79 \mathrm{mmol} / \mathrm{L}(3,79 \mathrm{mEq} / \mathrm{L})$ \\
\hline Cloro & Potenciometría indirecta & $100 \mathrm{mmol} / \mathrm{L}(100 \mathrm{mEq} / \mathrm{L})$ \\
\hline Calcio & o-cresolftaleìna complexona & $1,91 \mathrm{mmol} / \mathrm{L}(7,62 \mathrm{mg} / \mathrm{dL})$ \\
\hline Fósforo & Fosfomolibdato UV & $1,13 \mathrm{mmol} / \mathrm{L}(3,50 \mathrm{mg} / \mathrm{dL})$ \\
\hline Magnesio & Azul de xilidilo & 0,76 mmol/L (1,86 mg/dL) \\
\hline LDH & Lactato/NAD & $235 \mathrm{U} / \mathrm{L}$ \\
\hline CK & IFCC, activado con NAC & $182 \mathrm{U} / \mathrm{L}$ \\
\hline AST & IFCC modificado & $42 \mathrm{U} / \mathrm{L}$ \\
\hline ALT & IFCC modificado & $32,5 \mathrm{U} / \mathrm{L}$ \\
\hline FAL & IFCC modificado & $127,5 \mathrm{U} / \mathrm{L}$ \\
\hline GGT & IFCC modificado & $123,5 \mathrm{U} / \mathrm{L}$ \\
\hline Amilasa & pNPG7 bloqueado con etilideno & $93,5 \mathrm{U} / \mathrm{L}$ \\
\hline Lipasa & Cinética colorimétrica & $45,5 \mathrm{U} / \mathrm{L}$ \\
\hline
\end{tabular}

* GLDH: glutamato deshidrogenasa, CHOD: colesterol-oxidasa, GPO: glicerol fosfato oxidasa, UV: ultravioleta, LDH: lactato deshidrogenasa, CK: creatina cinasa, IFCC: Federación Internacional de Química Clínica,

NAC:N-acetil-L-cisteina, AST: aspartato aminotransferasa, ALT: alanina aminotransferasa, FAL: fosfatasa alcalina, GGT: gammaglutamiltransferasa, pNPG7: p-nitrofenil-maltoheptaósido. 
dores de Siemens ${ }^{\circledR}$. Para la medición de hemoglobina se utilizó un contador de células sanguíneas SYSMEX XE-2100 de Roche Diagnostics ${ }^{\circledR}$, mediante el método SLS (sulfato láurico de sodio).

Ambos analizadores fueron calibrados previamente de acuerdo a las recomendaciones del fabricante. El programa de control de calidad interno incluyó la evaluación de sueros controles $\mathrm{BIO}-\mathrm{RAD}^{\circledR}$ de 2 niveles de decisión, que se procesaron diariamente, y una muestra quincenal de control de calidad externo internacional (RIQAS).

En la tabla 1 se muestra los constituyentes analizados, los métodos en los que se basan las mediciones de los distintos analitos y la concentración sérica basal (sin interferente) de los constituyentes en estudio.

El estudio es descriptivo comparativo, donde se compara el valor medido de la magnitud en una muestra sin interferente con los valores obtenidos cuando se adicionan a la muestra concentraciones conocidas del interferente. Para lo cual se ha seguido el protocolo de la Comisión de Metrología y Sistemas Analíticos de la Sociedad Española de Química Clínica ${ }^{(8)}$.

Para la preparación del hemolizado, se centrifugó a $1200 \mathrm{~g}$ durante $10 \mathrm{mi}$ nutos $5 \mathrm{~mL}$ de sangre total heparinizada. Posteriormente se eliminó el plasma y se reemplazó con $10 \mathrm{~mL}$ de solución salina $(\mathrm{NaCl} 154 \mathrm{mmol} / \mathrm{L})$; se invirtió el tubo varias veces, se centrifugó por 10 minutos a $1200 \mathrm{x}$ g y se decantó el sobrenadante. Este proceso de lavado con solución salina se repitió 2 veces más. En el último lavado se desechó el sobrenadante y se adicionó al paquete globular 2,5 $\mathrm{mL}$ de agua desionizada. Se mezcló invirtiendo el tubo varias veces. La solución así preparada fue congelada a $-20 \stackrel{\circ}{\circ}$ por 13 horas, al cabo de lo cual se descongeló, agitó y centrifugó 30 minutos a 1200 x g. Se descartó el sedimento (eliminación de estromas celulares) y se cuantificó en el sobrenadante la concentración de hemoglobina libre por el método de sulfato láurico de sodio. La concentración de hemoglobina de la solución de partida fue $10,5 \mathrm{~g} / \mathrm{dL}$.

Para la preparación del suero base, se recolectó $25 \mathrm{~mL}$ de un pool de sueros libre de hemólisis, lipemia o ictericia, proveniente de sujetos voluntarios de ambos sexos, sin importar la edad, y sin patologías conocidas.

Para conseguir varios grados de hemólisis, se añadió cantidades crecientes del hemolizado a siete diferentes alícuotas del pool de sueros (suero base). En esta preparación, la mezcla libre de hemolizado se igualó en volumen a las alícuotas con interferente, utilizando agua destilada. Se obtuvo siete muestras con concentraciones crecientes de hemoglobina y una basal sin interferente.

Se pudo medir la hemoglobina en las tres últimas diluciones $\left(6^{a}, 7^{a}, 8^{a}\right)$ y se determinó la concentración de hemoglobina teórica en las soluciones $1^{\mathrm{a}}, 2^{\mathrm{a}}$, $3^{\mathrm{a}}, 4^{\mathrm{a}}$ y $5^{\mathrm{a}}$, ya que estos valores estuvieron por debajo del límite de detección del analizador hematológico. Las concentraciones resultantes de las alícuotas fueron: $1^{\text {a }} 0 \mathrm{~g} / \mathrm{L}, 2^{\mathrm{a}} 0,26 \mathrm{~g} / \mathrm{L}, 3^{\mathrm{a}} 0,53$ $\mathrm{g} / \mathrm{L}, 4^{\mathrm{a}} 1,05 \mathrm{~g} / \mathrm{L}, 5^{\text {a }} 2,10 \mathrm{~g} / \mathrm{L}, 6^{\mathrm{a}} 3,25 \mathrm{~g} / \mathrm{L}$, $7^{\text {a }} 4,30 \mathrm{~g} / \mathrm{L}$ y $8^{\text {a }} 5,25 \mathrm{~g} / \mathrm{L}$ (figura 1 ).

Cada una de las diluciones se analizó por duplicado de forma independiente, y pudo determinarse así el porcentaje de variación en la concentración de cada analito ensayado en función del incremento en el grado de hemólisis de las muestras.
La evaluación de las interferencias se realizó mediante el método de Glick y col ${ }^{(9)}$, expresándose los resultados como un porcentaje del resultado original. Para ello, se hace una representación gráfica de esta relación mediante un interferograma, en el que se representa $\left(\mathrm{C} / \mathrm{C}_{\mathrm{O}}\right) \times 100$ frente a la concentración de hemoglobina de cada alícuota, donde $\left(\mathrm{C}_{\mathrm{O}}\right)$ es la concentración inicial sin interferente y (C) la concentración medida experimentalmente del constituyente en estudio. Las interferencias se compararon con las actuales especificaciones de calidad analítica para el máximo error sistemático deseable (MESD), derivada de la variación biológica intraindividual. Se consideró a la interferencia como clínicamente relevante cuando era superior al MESD ${ }^{(10,11)}$.

Para el caso puntual de la bilirrubina total, esta se trata de una variable que primero disminuye su valor y luego, a medida que aumenta la concentración del interferente, aumenta. Es decir, existe una relación de no linealidad, lo que indica que la interferencia en este caso depende no solo de la cantidad de hemoglobina sino también de la concentración de bilirrubina. Para tal efecto se procedió a realizar un ensayo, basado en el modelo de Kroll ${ }^{(12)}$, que utiliza una regresión múltiple con tres variables independientes: la concentración del constituyente, la concentración del interferente y el producto de los dos o la interacción constituyenteinterferente:

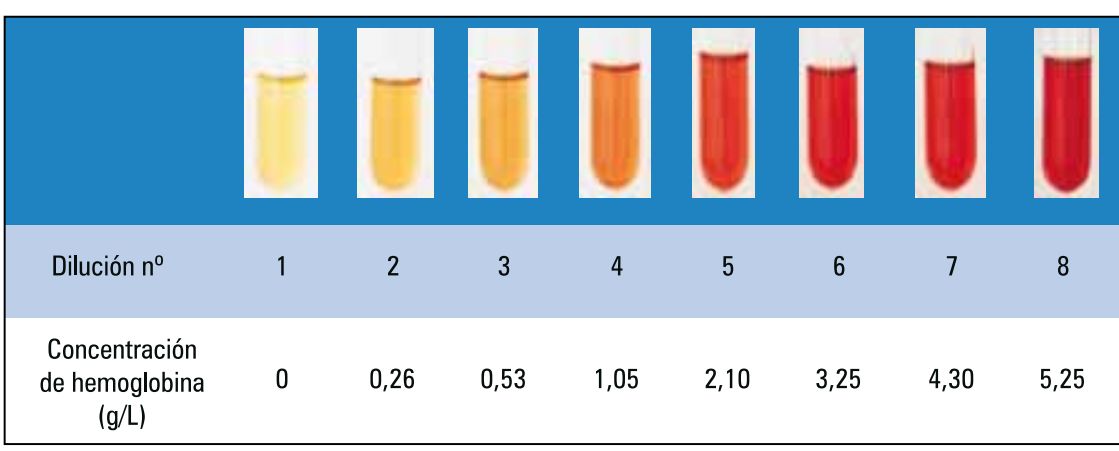

Figura 1. Alícuotas con cantidades crecientes del interferente (hemoglobina). 


$$
\mathrm{C}=\beta_{0}+\beta_{1} \mathrm{C}_{\mathrm{O}}+\beta_{2} \mathrm{I}+\beta_{3} \mathrm{C}_{\mathrm{O}} \mathrm{I}
$$

Donde $\mathrm{C}_{\mathrm{O}}=$ concentración inicial o teórica del constituyente en estudio, $\mathrm{C}=$ concentración experimental o medida, e I = concentración del interferente; el coeficiente $\beta_{0}$ representa el intercepto de la regresión múltiple y ha de ser próximo a cero; el coeficiente $\beta_{1}$ indica si el método es o no válido para dicho analito y ha de ser próximo a la unidad. Los coeficientes $\beta_{2}$ y $\beta_{3}$ representan la interferencia independiente y dependiente de la concentración del constituyente, respectivamente; el valor de cada uno de estos coeficientes está relacionado con la magnitud de la interferencia y su signo con la dirección de la interferencia, ya sea positiva o negativa.

El diseño del experimento para este modelo es una matriz ortogonal con concentraciones progresivamente crecientes del constituyente $(0,2 \mathrm{mg} / \mathrm{dL}$, $0,5 \mathrm{mg} / \mathrm{dL}, 1,0 \mathrm{mg} / \mathrm{dL}$ y $2 \mathrm{mg} / \mathrm{dL}$ de bilirrubina) y del interferente $(0 \mathrm{~g} / \mathrm{L}, 0,26$ $\mathrm{g} / \mathrm{L}, 0,53 \mathrm{~g} / \mathrm{L}, 1,05 \mathrm{~g} / \mathrm{L}, 2,1 \mathrm{~g} / \mathrm{L}, 3,25$ $\mathrm{g} / \mathrm{L}, 4,3 \mathrm{~g} / \mathrm{L}, 5,25 \mathrm{~g} / \mathrm{L}$ de hemoglobina). Es decir, se preparó 32 muestras diferentes, logrando conformar una matriz de cuatro concentraciones del constituyente y ocho concentraciones de hemoglobina.
Si los coeficientes $\beta_{2}$ o $\beta_{3}$ son distintos de cero, existe interferencia independiente o dependiente de la concentración del constituyente respectivamente. En algunos casos, ambos coeficientes son distintos de cero debido a una interferencia mixta dependiente e independiente de la concentración del constituyente. Si dichos coeficientes no son distintos de cero, no existen tales interferencias.

Con relación al análisis estadístico, se determinó el promedio y la imprecisión (coeficiente de variación) de los duplicados de la concentración de los constituyentes estudiados para cada

Tabla 2. Porcentaje relativo de desviación de la concentración del constituyente con respecto al resultado inicial por influencia de la hemólisis y especificaciones para el máximo error sistemático deseable.

\begin{tabular}{|c|c|c|c|c|c|c|c|c|c|}
\hline \multirow{2}{*}{ Constituyente } & \multirow{2}{*}{$\begin{array}{l}\text { Error sistemático } \\
\text { deseable } \%(+/-)\end{array}$} & \multicolumn{8}{|c|}{ Hemoglobina ( $g / \mathrm{L})$} \\
\hline & & 0,0 & 0,26 & 0,53 & 1,05 & 2,10 & 3,25 & 4,30 & 5,25 \\
\hline Glucosa* & 2,34 & 0,0 & 0,4 & 1,3 & 1,3 & 1,7 & 1,7 & 2,5 & 3,8 \\
\hline Urea & 5,57 & 0,0 & 0,0 & 0,0 & 0,0 & 0,0 & 0,0 & 1,5 & 1,5 \\
\hline Creatinina & 3,96 & 0,0 & $-0,3$ & $-0,3$ & $-0,3$ & $-0,3$ & 0,3 & 0,3 & 0,7 \\
\hline Ácido úrico & 4,87 & 0,0 & $-1,2$ & $-1,2$ & $-3,5$ & $-3,5$ & $-4,7$ & $-4,7$ & $-4,7$ \\
\hline Proteínas* & 1,36 & 0,0 & 0.5 & 0.3 & 0,9 & 1,6 & 2,8 & 5,2 & 6,1 \\
\hline Albúmina* & 1,43 & 0,0 & 0,4 & 0,5 & 0,9 & 1,3 & 3,8 & 5,9 & 7,5 \\
\hline Bilirrubina total & 8,95 & 0,0 & $-5,0$ & $-5,8$ & $-8,3$ & $-0,8$ & 0,0 & 0,8 & 1,65 \\
\hline Colesterol total ${ }^{*}$ & 4,10 & 0,0 & 1,8 & 1,8 & 2,2 & 5,1 & 5,1 & 6,9 & 8,3 \\
\hline HDL colesterol & 5,61 & 0,0 & 0,0 & 0,0 & 0,0 & 0,0 & 0,0 & 0,0 & 0,0 \\
\hline LDL colesterol & 5,46 & 0,0 & 0,6 & 0,0 & 0,6 & 2,3 & 2,3 & 3,4 & 3,4 \\
\hline Triglicéridos & 9,57 & 0,0 & 0,4 & 1,5 & 2,6 & 4,4 & 4,7 & 6,6 & 7,7 \\
\hline Sodio* & 0,23 & 0,0 & 0,0 & 0,0 & 0,0 & $-0,38$ & $-0,38$ & $-0,38$ & $-0,38$ \\
\hline Potasio* & 1,81 & 0,0 & 2,1 & 5,3 & 9,3 & 18,0 & 25,5 & 32,0 & 41,1 \\
\hline Cloro* & 0,47 & 0.0 & 0,0 & 0,0 & $-1,0$ & $-0,8$ & $-0,5$ & $-0,5$ & $-0,5$ \\
\hline Calcio & 0,82 & 0,0 & 0,2 & 0,2 & 0,1 & 0,5 & 0,0 & 0,7 & 0,2 \\
\hline Fósforo* & 3,38 & 0,0 & 0,9 & 1,6 & 2,7 & 4,0 & 6,6 & 8,6 & 10,9 \\
\hline Magnesio* & 1,84 & 0,0 & 1,6 & 1,1 & 1,9 & 1,9 & 3,8 & 7,5 & 5,4 \\
\hline $\mathrm{LDH}^{*}$ & 4,26 & 0,0 & 16,4 & 32,3 & 58,3 & 112 & 156 & 192 & 240 \\
\hline $\mathrm{CK}^{*}$ & 11,51 & 0,0 & 2,8 & 6,3 & 10,5 & 20,7 & 31,4 & 39,7 & 46,8 \\
\hline$A S T^{*}$ & 6,54 & 0,0 & 2,4 & 10,7 & 20,2 & 33,3 & 50,0 & 63,1 & 82,1 \\
\hline $\mathrm{ALT}^{*}$ & 11,48 & 0,0 & 4,6 & 9,2 & 10,8 & 12,3 & 20,0 & 20,0 & 23,1 \\
\hline $\mathrm{FAL}^{*}$ & 6,72 & 0,0 & $-5,1$ & $-5,1$ & $-8,2$ & $-11,0$ & $-15,3$ & $-17,6$ & $-25,5$ \\
\hline GGT & 11,06 & 0,0 & $-1,2$ & $-2,0$ & $-3,2$ & $-2,0$ & $-1,2$ & $-2,4$ & $-4,0$ \\
\hline$\alpha$-Amilasa* & 7,40 & 0,0 & 1,1 & 0,5 & $-0,53$ & $-1,6$ & $-3,2$ & $-4,8$ & $-9,1$ \\
\hline Lipasa* & 11,31 & 0,0 & 0,0 & 1,1 & 5,5 & 12,1 & 18,7 & 25,3 & 30,8 \\
\hline
\end{tabular}

Los resultados son comparados con las especificaciones de inexactitud deseable, los que exceden dichas especificaciones están marcados en negrita y cursiva.

$\left({ }^{*}\right)$ Constituyentes en las que se encuentra interferencia. 

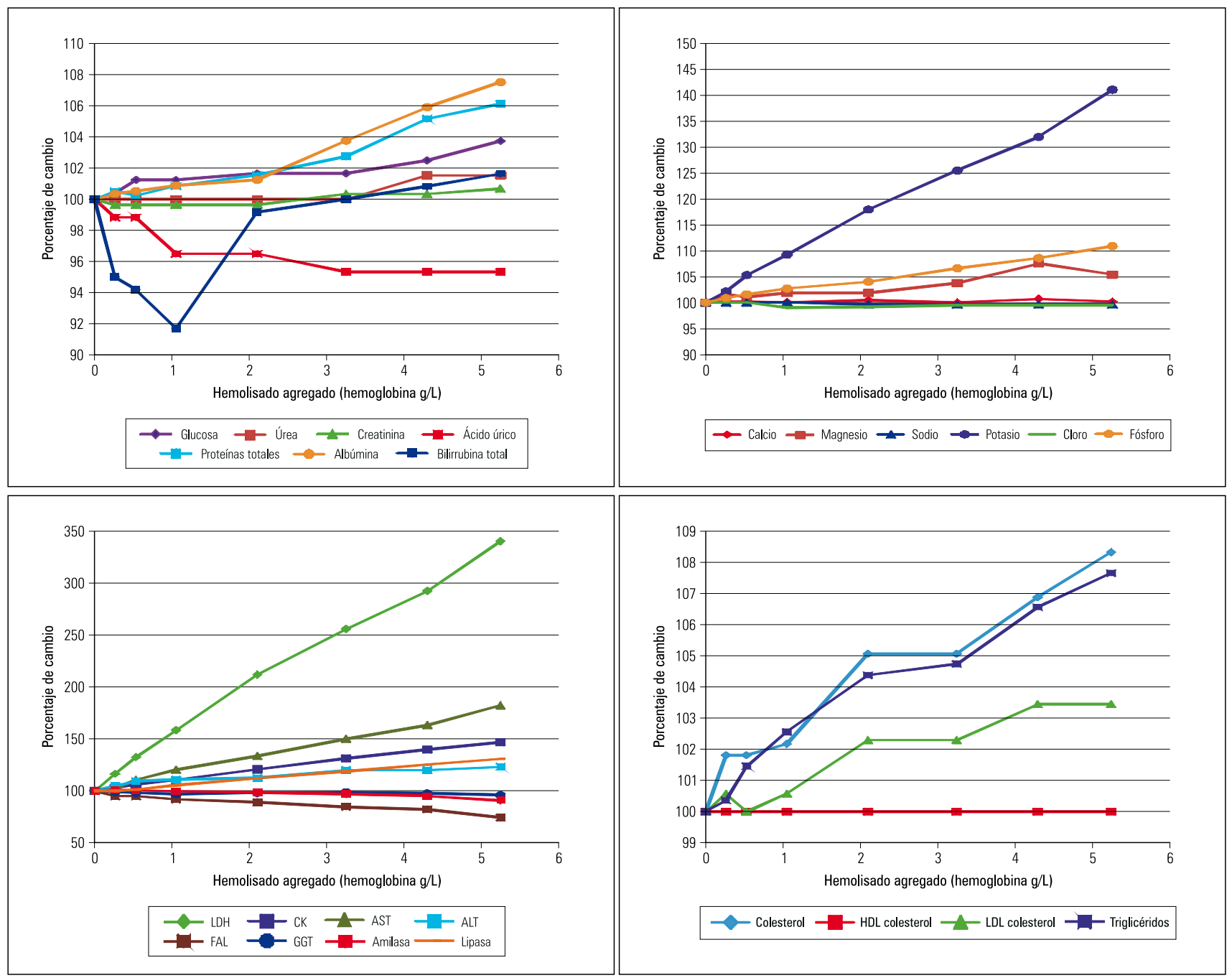

Figura 2. Interferogramas donde se muestra el efecto del agregado de cantidades crecientes del interferente sobre la concentración original (porcentaje de cambio) de los 25 constituyentes en estudio.

alícuota. Para el caso particular de la bilirrubina, se realizó el análisis de regresión múltiple, el cálculo de los coeficientes de dicha regresión y la probabilidad de que los coeficientes fueran significativamente diferentes de cero, para $\mathrm{p}<0,05$. Para el análisis estadístico se empleó el software SPSS versión 21 (SPSS Inc., Chicago, IL, EE. UU.).

\section{RESULTADOS}

Utilizando el criterio de máximo error sistemático deseable, basados en estudios de variabilidad biológica ${ }^{(10,11)}$, en el presente estudio se encontró interferencia clínicamente relevante en 16 constituyentes de los 25 estudiados.

Los constituyentes urea, creatinina, ácido úrico, bilirrubina total, colesterol HDL, colesterol LDL, triglicéridos, calcio y gammaglutamil transferasa no presentaron interferencia debida a hemólisis en todas las alícuotas con concentraciones crecientes de hemoglobina empleadas en el ensayo.

Se observó interferencia para glucosa, proteínas, albumina, colesterol, potasio, fósforo, magnesio, deshidrogenasa láctica, creatinfosfoquinasa, aspartato aminotransferasa, alanino aminotransferasa, lipasa, sodio, cloro, fosfatasa alcalina y amilasa.

La imprecisión expresada en términos de coeficiente de variación presentó un valor mínimo de $0 \%$ y máximo de 3,6\% para todos los constituyentes analizados por duplicado.

En la tabla 2 se expone los porcentajes relativos de desviación de la concentración del constituyente con respecto al resultado inicial, las especificaciones de calidad analítica para el máximo error sistemático deseable y las alícuotas donde se detecta la in- 
terferencia clínicamente relevante para cada uno de los constituyentes.

En la figura 2 se representa los interferogramas ${ }^{(9)}$, donde se aprecia el efecto de la adición de cantidades crecientes del interferente (hemoglobina) sobre la concentración de los analitos estudiados.

Para el caso de la bilirrubina, se obtuvo la siguiente ecuación de regresión múltiple:

$$
\begin{aligned}
\mathrm{C}= & -0,014+0,944 \mathrm{C}_{\mathrm{O}}-0,06 \mathrm{I}+ \\
& 0,04 \mathrm{C}_{\mathrm{O}} \mathrm{I}
\end{aligned}
$$

En nuestro estudio, el valor del coeficiente $\beta_{0}=-0,014$ estuvo próximo a cero, mientras que el coeficiente $\beta_{1}=$ 0,944 se acercó a la unidad, por lo que puede decirse que este método es válido para la evaluación de esta técnica analítica. Los coeficientes $\beta_{2}$ y $\beta_{3}$ no resultaron significativamente distintos de cero $(p>0,05)$, lo que confirma la no interferencia de hemoglobina hasta una concentración de $5,25 \mathrm{~g} / \mathrm{L}$ para la determinación de bilirrubina total. Resultados que son detallados en la tabla 3.

\section{DISCUSIÓN}

La interferencia analítica por hemólisis es un problema que afecta a todos los laboratorios. Siempre se cuestiona la exactitud de los resultados cuando se analiza este tipo de muestras. La solución no está en solo añadir comentarios como 'muestra hemolizada' a los reportes de laboratorio; es importante también conocer la cantidad y el signo posible de dicha interferencia.

Los primeros estudios publicados sobre el efecto de la hemólisis consideraban como interferencia significativa un $10 \%$ de variación sobre el resultado de la muestra sin interferente. Este criterio, utilizado por muchos fabricantes en sus estudios de evaluación, es más permisivo en las magnitudes con pequeña variabilidad biológica y puede ser menos permisivo en aquellas con elevada variabilidad intraindividual o interindividual ${ }^{(1,6)}$.

Tabla 3. Análisis de los coeficientes de regresión múltiple resultantes de la interferencia de la hemoglobina con bilirrubina total $\left(R^{2}=0,994\right)$.

\begin{tabular}{cccc} 
Variable & Coeficiente & Valor del coeficiente & Nivel $p^{*}$ \\
Intercepto & $\beta_{0}$ & $-0,014$ & 0,578 \\
Bilirrubina total & $\beta_{1}$ & 0,944 & 0,000 \\
Interferente (hemoglobina) & $\beta_{2}$ & $-0,006$ & 0,498 \\
Bilirrubina total - Interferente & $\beta_{3}$ & 0,004 & 0,650 \\
\hline
\end{tabular}

* Valores de $p<0,05$ son considerados significativamente diferentes de cero.

Nuestros resultados indican que, si hubiéramos utilizado el criterio anteriormente mencionado, algunos constituyentes no hubieran sido detectados como sensibles a la presencia del interferente. Lo que sugiere que la significación del efecto de la hemólisis debe establecerse en cada laboratorio y para cada magnitud de acuerdo con sus propias especificaciones de calidad.

Ante la presencia de hemólisis con una concentración de hemoglobina igual o mayor a $0,53 \mathrm{~g} / \mathrm{L}$, los resultados de potasio, deshidrogenasa láctica y aspartato aminotransferasa se vieron afectados, lo que se traduce en una pérdida de fiabilidad en los resultados obtenidos para los analitos, inclusive cuando se presenta hemólisis ligera. Diversos estudios han encontrado que dicha interferencia es debida a las mayores concentraciones de estos constituyentes en el interior del hematíe que en el suero ${ }^{(5,14,15)}$.

En cuanto al colesterol, que usa la reacción de Trinder como reacción indicadora, hemos obtenido interferencia al igual que otros estudios ${ }^{(14,15)}$, lo cual parece lógico dado las propiedades seudoperoxidasa de la hemoglobina.

En el caso de los triglicéridos y el ácido úrico que también usan la reacción de Trinder como reacción indicadora, no hemos obtenido interferencia significativa, resultado que concuerda con Lippi y Caballero ${ }^{(5,14)}$, pero que discrepan con Steen y Castaño, que obtuvieron interferencia para ambos constituyentes ${ }^{(15,16)}$.

Para el caso de las lipoproteínas de alta densidad (HDL) y de baja densi- dad (LDL) determinadas mediante el método directo, se advirtió interferencia nula para ambos casos, resultado similar a lo citado por Aguilar ${ }^{(12)}$.

En similitud con los resultados de otros estudios ${ }^{(5,14,15,17)}$, encontramos interferencia debida a la hemólisis en la medición de la actividad de la enzima fosfatasa alcalina, que se detecta a una concentración igual o mayor de 1,05 $\mathrm{g} / \mathrm{L}$ de hemoglobina, atribuida a una interferencia espectral.

Para el caso concreto de la amilasa, esta presentó interferencia en la última alícuota, correspondiente a una concentración de hemoglobina de $5,25 \mathrm{~g} / \mathrm{L}$, resultado que no coincide con Aguilar, quien determinó interferencia no significativa para dicho analito en el autoanalizador Sistema Modular Roche/ Hitachi SWA. Sin embargo, en este estudio se utilizó como última alícuota una concentración de hemoglobina de $5,11 \mathrm{~g} / \mathrm{L}^{(13)}$.

En el caso de los electrolitos, el único que presentó efecto nulo por hemólisis fue el calcio, resultado que coincide con otros estudios ${ }^{(5,13-15,17)}$.

Nuestros resultados para la albúmina y las proteínas coinciden con los de Steen ${ }^{(15)}$, pero no con los publicados por Aguilar (13). Hay que considerar que el principio del método para dosar proteínas se basa en la formación de un complejo morado como consecuencia de la interacción de los enlaces peptídicos de las proteínas con los iones de cobre del reactivo de Biuret, que se mide a 545 nanómetros (nm) como reacción de punto final, longitud de onda que coincide con los puntos de máxima 
absorbancia de la hemoglobina, lo cual parece ser la causa para dicha interferencia.

Al igual que otros estudios ${ }^{(5,13,14)}$, encontramos una sobreestimación en los resultados de actividad de la enzima creatinfosfoquinasa, interferencia que ha sido atribuida a la liberación de la adenilatoquinasa intracelular, como consecuencia de la hemólisis.

La gammaglutamiltransferasa no se vio afectada por la hemólisis en todas las alícuotas empleadas en el estudio, resultado semejante a lo obtenido por Aguilar, quien empleó la misma metodología pero diferente autoanalizador ${ }^{(13)}$.

Destaca en nuestros resultados la interferencia para glucosa a concentraciones de hemoglobina igual o mayor de 4,30 g/L, dato que discrepa con Aguilar y Caballero ${ }^{(13,14)}$, quienes en diferentes analizadores no encontraron interferencia, a pesar que en estos dos estudios emplearon la misma metodología (hexocinasa).

Para la determinación de creatinina se usó la técnica enzimática de creatininasa, la cual presentó interferencia nula para hemólisis en todas las diluciones de hemoglobina empleadas en nuestro estudio, resultados que concuerdan con Aguilar, quien utilizó la misma metodología pero diferente analizador ${ }^{(13)}$. Es importante señalar que Murray reporta interferencia a partir de una concentración de hemoglobina de $1 \mathrm{~g} / \mathrm{L}$ para esta determinación, utilizando la metodología de Jaffé cinético ${ }^{(7,19)}$.

El estudio de valoración de interferencia por hemólisis debe realizarse con una muestra que tenga una concentración del analito próxima a los valores de decisión clínica. En algunos casos, el efecto de la interferencia no solo depende de la concentración de la sustancia interferente, sino también de la concentración del constituyente, como fue el caso de la bilirrubina. En nuestro estudio nos ha resultado imposible obtener un pool de sueros cuyas concentraciones estén todas próximas a los valores de decisión clínica.
La interferencia espectral por hemólisis se produce cuando el espectro de absorción del interferente y la del cromógeno producido en la reacción se solapan; muchos instrumentos de uso corriente emplean diferentes técnicas para la corrección de las interferencias espectrales. Estas técnicas involucran el uso de un blanco de muestra, el análisis bicromáticos o mediante la corrección de Allen ${ }^{(7)}$, todo esto sumado a las diferentes metodologías o reactivos que se emplean para medir los diferentes constituyentes, además del empleo de diferentes criterios para establecer el límite de error máximo admisible para establecer interferencia significativa, pueden ser causas de la discordancia de resultados en los diferentes estudios para este tipo de interferencia.

Es interesante notar que metodologías teóricamente idénticas o muy semejantes brindan resultados distintos ante la presencia de hemólisis, lo que nos indicaría que el análisis de interferencia aquí realizado no puede ser generalizado para otros reactivos o equipos. Es recomendable que cada laboratorio investigue los efectos de dicha interferencia empleando sus propios reactivos, métodos o instrumentos.

La mayoría de los actuales autoanalizadores tiene la capacidad de detectar la hemólisis en las muestras de suero mediante índices séricos que se correlacionan linealmente con las concentraciones del interferente. Los índices pueden ser usados para detectar la interferencia generando tablas de tolerancia, con índices de decisión que indican cuándo hay una interferencia. En nuestro medio, a pesar de contar con estos equipos, no se hace uso de esta tecnología por desconocimiento o por la excusa de consumo de tiempo de proceso del analizador y necesidad de reactivos específicos en algún caso ${ }^{(6,8,13)}$.

Podemos concluir que las muestras hemolizadas son un problema importante en todos los laboratorios. Las principales causas que la originan son la extracción o manejo de la muestra de sangre de forma inadecuada, especialmente en áreas de alta presión asistencial como en las unidades de cuidados intensivos y los servicios de emergencia.

Los laboratorios deben asegurar la detección de la hemólisis y tener establecidas las acciones que se van a tomar frente a estas muestras.

\section{AGRADECIMIENTOS}

Al Licenciado Tecnólogo Médico Arturo Arzapalo Poma por su tiempo y aportes durante el desarrollo del presente trabajo.

\section{REFERENCIAS BIBLIOGRÁFICAS}

1. Castaño JL. Estudio de las interferencias analiticas endógenas en química clínica. Quim Clin 1994;13:84-92.

2. Castaño JL. Criterios para la valoración de la significación analítica y clínica de las interferencias en bioquímica clínica. Quím Clín. 1995;14:107-9.

3. Clinical and Laboratory Standards Institute. Interference Testing in Clinical Chemistry; Approved Guideline-Second Edition. EP7-A2. USA 2010.

4. Lippi G, Blanckaert N, Bonini P, Green S, Kitchen S, Palicka V, et al. Haemolysis: an overview of the leading cause of unsuitable specimens in clinical laboratories. Clin Chem Lab Med. 2008;46:764-72. doi: 10.1515/CCLM.2008.

5. Lippi G, Salvagno GL, Montagnana M, Brocco G, Guidi GC. Influence of hemolysis on routine clinical chemistry testing. Clin Chem Lab Med. 2006;44:311-6.

6. Gómez Rioja R, Alsina Kirchner MJ, Alvarez Funes V, Barba Meseguer N, Cortés Rius M, 345 Llopis Díaz MA, Martínez Bru C. Hemólisis en las muestras para diagnóstico. Rev Lab Clin. 2009;2(4):18595. doi: 10.1016/j.labcli.2009.08.002

7. Kaplan L, Pesce A. Interferencias en el análisis espectral. En: Kaplan L, Pesce A. Quimica Clinica, teoria, análisis y correlación. Buenos Aires: Ed. Médica Panamericana; 1988:1163-76.

8. Sociedad Española de Bioquímica Clínica y Patología Molecular. Procedimiento para el estudio de la interferencia por hemólisis, bilirrubina y turbidez y para la verificación de los indices de hemólisis, ictericia y lipemia. Comisión de Metrologia y Sistemas Analíticos. Documento Técnico 2013.

9. Glick MR, Ryder KW, Jackson SA. Graphical comparisons of interferences in clinical chemistry instrumentation. Clin Chem. 1986;32:470-5.

10. Ricós C, García Lario JV, Álvarez V, Cava F, Doménech MV, Hernández A, et al. Biological variation database. The 2014 update. [Consultado 8/7/2014]. Disponible en: http://www. westgard. com/biodatabase1.htm.

11. Sociedad Española de Bioquímica Clínica y Patología Molecular. Comité de garantía de la Calidad y Acreditación de Laboratorios. Comisión de calidad Analítica. Base de datos De variación biológica. Actualización del año 2014: Disponible en http:// www.seqc.es/es/Sociedad/51/102. 
12. Castaño J, Ventura S. Recomendaciones para el estudio de las interferencias dependientes de la concentración del constituyente. Quim Clin 2001;20(4):257-60.

13. Garcia Aguilar GD, Pico Picos MA, Quintana Hidalgo L, Cabrera Argany A, Lorenzo Medina M, Aguilar Doreste JA. Utilidad de los índices séricos para la valoración de las interferencias causadas por la hemólisis y la bilirrubina en la medición de distintos constituyentes bioquímicos. Quim Clin. 2007;26:196-201.

14. Caballero Sarmiento R. Estudio de las interferencias producidas por la hemólisis en la medición de 18 constituyentes séricos en un ADVIA 2400. Quím Clin. 2007;26:20-2.

15. Steen G, Vermeer HJ, Naus AJ, Goevaerts B, Agricola PT, Schoenmakers $\mathrm{CH}$. Multicenter evaluation of the interference of hemoglobin, bilirubin and lipids on Synchron LX-20 assays. Clin Chem Lab Med. 2006;44:413-9.

16. Castaño JL, Araquistain JL Interferencias causadas por la bilirrubina, hemoglobina y hemólisis en la determinación de 15 constituyentes séricos. Quìm Clín. 1989;8:47-55.

17. Ji JZ, Meng QH. Evaluation of the interference of hemoglobin, bilirubin, and lipids on Roche Cobas 6000 assays. Clin Chim Acta. 2011 Aug 17;412(1718):1550-3. doi: 10.1016/j.cca.2011.04.034.

18. Grafmeyer D, Bondon M, Manchon M, Levillain P. The influence of bilirubin, hemolysis and turbidity on 20 analytical tests performed on automatic analysers. Results of an interlaboratory study. Eur J Clin Chem Clin Biochem. 1995;33:31-52.

19. Murray RL. Creatinina. En Kaplan L, Pesce A, Quimica Clínica, teoria, análisis y correlación. Buenos Aires: Ed. Médica Panamericana; 1988.
Artículo recibido el 3 de marzo de 2015 y aceptado para publicación el 6 de junio de 2015.

Conflicto de intereses:

El autor declara no tener ningún conflicto de intereses.

\section{Correspondencia:}

ítalo Moisés Saldaña Orejón

Departamento de Patología Clínica, Servicio de Bioquímica, Hospital Nacional Edgardo Rebagliati Martins, EsSalud, Lima, Perú

imso_biochemical@yahoo.es 